\title{
Presencia de estructuras excepcionalmente preservadas en vías aéreas de Panochthus Burmeister (Xenarthra, Glyptodontidae) del Pleistoceno tardío de Argentina
}

\author{
Martín ZAMORANO
}

División de Paleontología de Vertebrados, Facultad de Ciencias Naturales y Museo, Universidad Nacional de La Plata. Paseo del Bosque s/n, 1900 La Plata. Argentina. CONICET. Cátedra de Zoología Vertebrados. marzamorano@fcnym.unlp.edu.ar

Zamorano, M. 2020. Presencia de estructuras excepcionalmente preservadas en vías aéreas de Panochthus Burmeister (Xenarthra, Glyptodontidae) del Pleistoceno tardío de Argentina [Presence of exceptionally preserved structures in the breathing areas of Panochthus Burmeister (Xenarthra, Glyptodontidae) from the Late Pleistocene of Argentina]. Spanish Journal of Palaeontology, 35 (2), 167-174.

Manuscript received 3 March 2020

Manuscript accepted 15 May 2020 https://doi.org/10.7203/sjp.35.2.18479

(C) Sociedad Española de Paleontología ISSN 2255-0550

\section{RESUMEN}

En esta contribución se presentan materiales excepcionalmente preservados correspondientes a las zonas laríngea y traqueal de las vías aéreas de gliptodóntidos (Xenarthra; Cingulata): dos de la zona laríngea, el cartílago tiroides (MPLK 0004) y el cartílago cricoides (MHM-P 87), y una de la zona traqueal, fragmentos de los anillos traqueales (MHM-P 87). Todos estos cartílagos están osificados y proceden del Pleistoceno tardío de Buenos Aires (Argentina). A su vez, se reconstruyen los músculos que involucran a los cartílagos mencionados. El material de estudio corresponde a dos especímenes, ambos previamente atribuidos a Panochthus sp. Panochthus Burmeister es uno de los Glyptodontidae más frecuentes y diversificados del Pleistoceno de América del Sur. El cartílago tiroides tiene una sección transversal en forma de U, en la porción anterior están las carillas para el hueso impar y en la posterior las carillas donde contacta el cricoide. Los 20 fragmentos de anillos traqueales principalmente varían entre 19 y $54 \mathrm{~mm}$ de largo y entre 6 y $8 \mathrm{~mm}$ de ancho. El cartílago cricoides tiene forma similar a la de un anillo traqueal, aunque es de mayor tamaño. La relación de tamaño entre los cartílagos estudiados verifica que las proporciones son las mismas que en otros xenartros. Las rugosidades en la lámina lateral del cartílago tiroides permiten concluir que la inserción del músculo cricotiroides es importante y el último cartílago

\begin{abstract}
In this contribution, exceptionally preserved materials related to the laryngeal and tracheal areas of the glyptodontids airways (Xenarthra; Cingulata) are presented: two of the laryngeal zone, the thyroid cartilage (MPLK 0004) as well as the cricoid cartilage (MHM-P 87), and one of the tracheal zone, fragments of the tracheal rings (MHM-P 87). All these cartilages are ossified and come from the Late Pleistocene of Buenos Aires (Argentina). In turn, the muscles that involve the aforementioned cartilage are reconstructed. The studied material corresponds to two different specimens attributed to Panochthus sp. in previous publications. Panochthus Burmeister is one of the most frequent and diversified Glyptodontidae of the Pleistocene in South American. The thyroid cartilage is U-shaped in section, in the anterior portion are the articular surfaces for the $\mathrm{V}$-bone and in the posterior part the veneers where the cricoid contacts. The 20 fragments of tracheal rings rang from 19 to $54 \mathrm{~mm}$ long and from 6 to $8 \mathrm{~mm}$ wide. The cricoid cartilage is similar in shape to a tracheal ring, although larger. The size relationship between the study cartilages verifies that the proportions are the same as in other xenarthros. The roughness in the lateral lamina of the thyroid cartilage allows us to conclude that the insertion of the cricothyroid muscle is important and the last cartilage moves significantly towards to the skull. Studies like this
\end{abstract}


se desplaza significativamente hacia el cráneo. Estudios como este generarán nuevos conocimientos sobre la paleobiología de la megafauna sudamericana.

Palabras claves: Mamíferos fósiles, cartílago tiroides, cartílago cricoides, cartílagos traqueales, América del Sur. will generate new knowledge about the palaeobiology of the South American mammal megafauna.

Keywords: Fossil mammals, thyroid cartilage, cricoid cartilage, tracheal cartilages, South America.

\section{INTRODUCCIÓN}

En los mamíferos, el aire atmosférico ingresa en los pulmones a través de las vías aéreas (König \& Liebich, 2005a). Según estos autores las vías aéreas que se encuentran en la región de la cabeza son llamadas vías aéreas superiores, en tanto que las que están desde la cabeza hasta llegar a los pulmones se denominan vías aéreas inferiores. La laringe, formada por seis cartílagos (cuneifome, corniculado, epiglótico, arítenoides, tiroides y cricoides), es el órgano que inicia el recorrido por las vías aéreas inferiores, contactando su extremo anterior con el basihiodes (ver Zamorano et al., 2018). Los cartílagos tiroides y cricoides son cartilaginosos hialinos que pueden osificarse (Sisson \& Grossman, 1982). La mayor parte del arítenoides también es de cartílago hialino, pero este último no suele osificarse (Sisson \& Grossman, 1959). El recorrido por las vías aéreas inferiores hacia los pulmones lo continúa la tráquea que se extiende desde el cartílago cricoides de la laringe hasta el mediastino medio, donde se bifurca en los bronquios principales (König \& Liebich, 2005a). El armazón de la tráquea está formado por placas de cartílago hialino en forma de C (anillos traqueales). Desde un punto de vista del desarrollo, el cartílago tiroides deriva del tejido mesenquimatoso del cuarto arco y el cartílago cricoides deriva del mesénquima relacionado con el sexto arco (Sisson \& Grossman, 1982), en tanto que los cartílagos traqueales se originan del mesodermo esplácnico y conforman el verdadero esqueleto traqueal (Acuña Navas et al., 2010).

Los xenartros son un particular grupo de mamíferos característico de la Región Neotropical, ampliamente representado en el registro fósil de América del Sur, tanto por su extensión temporal como por su frecuencia de registros (Scillato-Yané, 1977; Paula Couto, 1979; ScillatoYané \& Carlini, 1995). La familia Glyptodontidae constituye el grupo de xenartros acorazados cuyos representantes alcanzan grandes tamaños, a veces gigantesco (ScillatoYané \& Carlini, 1998; Fariña, 2001; Zamorano et al., 2014a), pudiendo superar los $2.300 \mathrm{~kg}$ (Soibelzon et al., 2012), y se registran desde el Eoceno medio hasta el Holoceno temprano (Scillato-Yané, 1976; Fernicola, 2008; Zamorano, 2013; Zurita et al., 2016). Panochthus Burmeister representa uno de los gliptodóntidos más abundantes y diversificados del Pleistoceno sudamericano, así como uno de los Cingulata de mayor tamaño (ver
Fariña, 2001; Zamorano et al., 2014a, 2014b). Junto con Glyptodon Owen son los gliptodóntidos de mayor distribución latitudinal y altitudinal en América del Sur (Zurita et al., 2009; Zamorano, 2012; Zurita et al., 2016; Zamorano \& Jara Almonte, 2018).

En la presente contribución se describen detalladamente y se figuran los cartílagos excepcionalmente preservados de las vías aéreas de Panochthus sp. Asimismo, se realiza una descripción comparativa de estos materiales y se reconstruyen los músculos que involucran a los cartílagos tiroides, cricoides y traqueal.

\section{MATERIALES Y PROCEDENCIAS}

Los ejemplares estudiados fueron asignados por Zamorano et al. (2018) a dos individuos pertenecientes a Panochthus sp., ambos ejemplares con material asociado que permitió su determinación: 1) MPLK 0004, cráneo muy completo con ambas ramas mandibulares, el aparato hioides completo con todos sus elementos pares e impares, el cartílago tiroides osificado y el escudete cefálico. Se encuentra depositado en el Museo Paleontológico Lucas Kraglievich, Marcos Paz, Buenos Aires, Argentina (MPLK). Fue recolectado en la Cantera Nicolás Vignogna III, Marcos Paz (3454'51" $\mathrm{S}-58^{\circ} 42^{\prime} 28^{\prime \prime}$ O) (Fig. 1a). El ejemplar proviene de sedimentos asignados a la Formación Luján datados en ca. $30 \mathrm{kyr}$ AP, Pleistoceno tardío (Gasparini et al., 2016), y fue descrito por Zamorano et al. (2018). 2) MHM-P 87, parte del cráneo con las mandíbulas fragmentadas, el hueso impar y fragmentos de los huesos pares del aparato hioides, cartílago cricoides y fragmentos de cartílagos traqueales osificados, ambos fémures, y ambos húmeros, tibia-fíbula y radio-ulna, varios elementos de los tarsos y carpos, tubo dorsal y lumbar, costillas cervicales, y la coraza dorsal casi completa. Se encuentra depositado en el Museo Histórico Municipal Alfredo Enrique Múlgura, General Belgrano, Buenos Aires, Argentina (MHM). Este material fue hallado en los márgenes del Río Salado, cerca de la Estancia La Invernada, General Belgrano (3545'5.52" S - 58 37'36" O) (Fig. 1b), en sedimentos atribuibles a la Formación Luján datados entre 14 y 12 kyr AP, Pleistoceno tardío (Scanferla et al., 2013). Los cartílagos mencionados fueron descriptos por Zamorano (2020). 


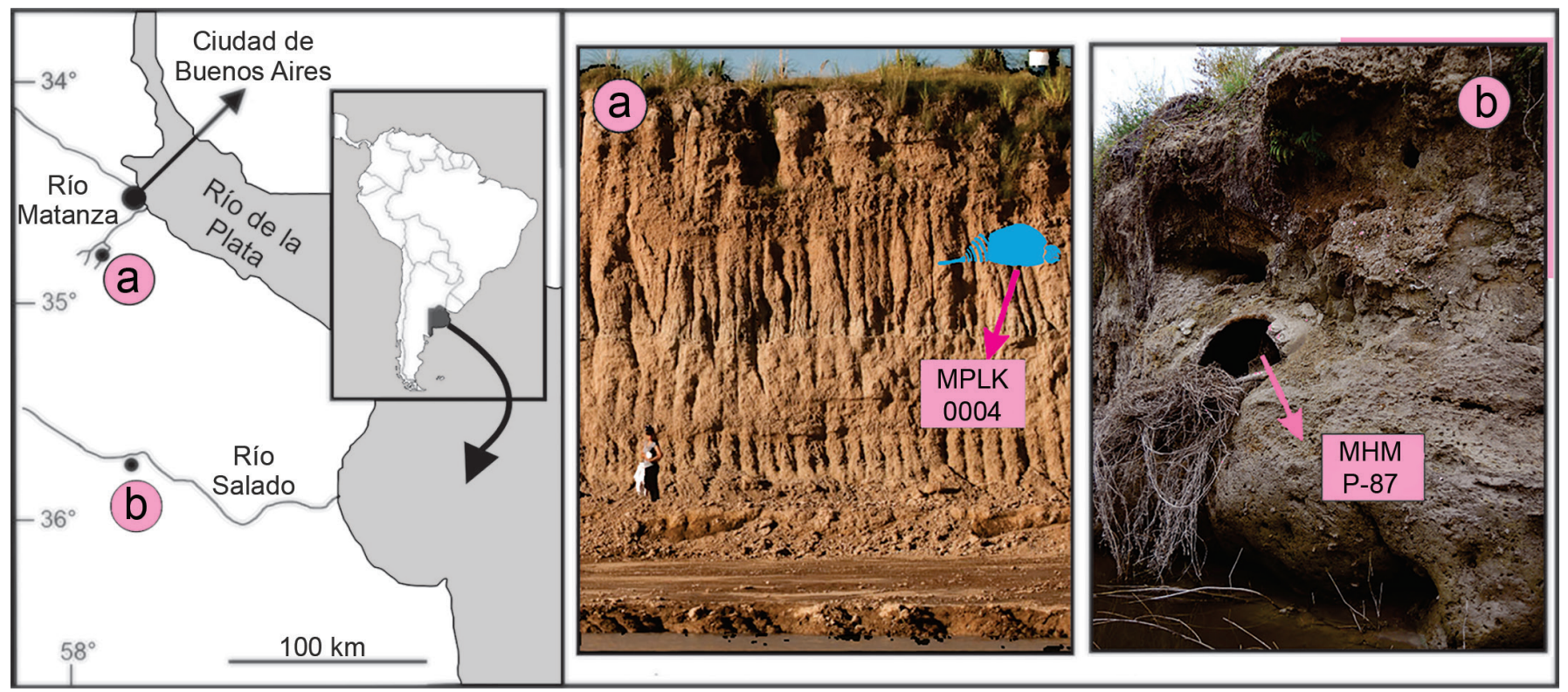

Figura 1. Ubicación geográfica de los materiales estudiados. a) “Cantera Nicolás Vignogna III”, Marcos Paz, Buenos Aires. b) Márgenes del Río Salado, General Belgrano, Buenos Aires.

Todas las medidas aportadas se expresan en milímetros $(\mathrm{mm})$, con un margen de error de 0,5 $\mathrm{mm}$. Las diferentes dimensiones medidas (Fig. 2) fueron tomadas con calibre manual y con cinta métrica.

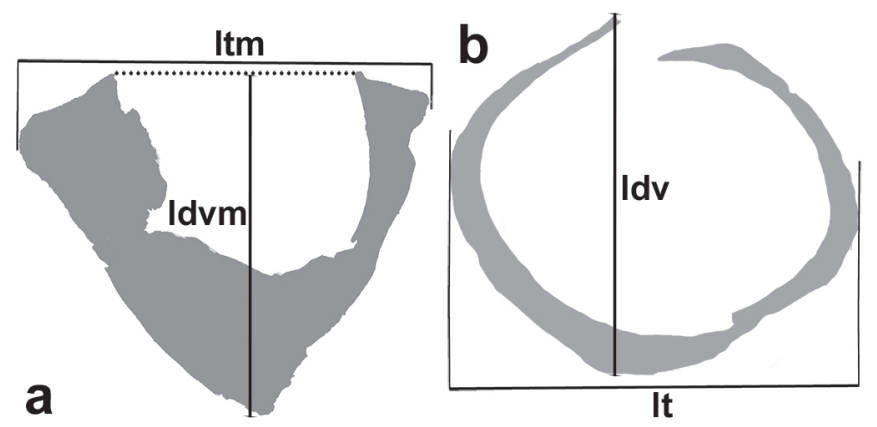

Figura 2. Medidas extraídas. a) Cartílago tiroides. b) Anillo traqueal. ldv, longitud dorsoventral; ldvm, longitud dorsolventral maxima; lt, longitud transversal; ltm, longitud transversal máxima.

\section{PALEONTOLOGÍA SISTEMÁTICA}

Magnorden XENARTHRA Cope, 1889

Orden CINGULATA Illiger, 1811

Suborden GLYPTODONTIA Ameghino, 1889

Superfamilia Glyptodontoidea Gray, 1869

Familia Glyptodontidae Gray, 1869
Género Panochthus Burmeister, 1866

Especie tipo Glyptotdon tuberculatus Owen, 1845

\section{Panochthus sp.}

\subsection{Descripción comparativa entre los materiales correspondientes a las vías aéreas}

\subsubsection{Cráneo MPLK 0004}

El cartílago tiroides está completo y es extremadamente frágil. Es el mayor cartílago de las vías aéreas. Se puede apreciar que, como en la mayoría de mamíferos vivientes, está constituido por dos láminas subcuadradas que se fusionan en su línea media. En vista transversal tiene una sección en forma de U. Dorsalmente, en vista anterior, se pueden apreciar las carillas donde contacta el hueso impar (V-bone), sobre el cual pivotea. En esta vista, ventralmente se ve una sutura en donde se encontraría la protuberancia tiroidea. En la parte posterior, dorsalmente se observan las carillas en donde contacta el cartílago cricoides. Ventralmente, en vista posterior, el extremo anterior se desvía ventralmente con respecto al extremo posterior (Fig. 3a).

\subsubsection{Cráneo MHM-P 87}

Hay tres fragmentos del cartílago cricoides de formas y tamaños variables, todos más anchos que los fragmentos 


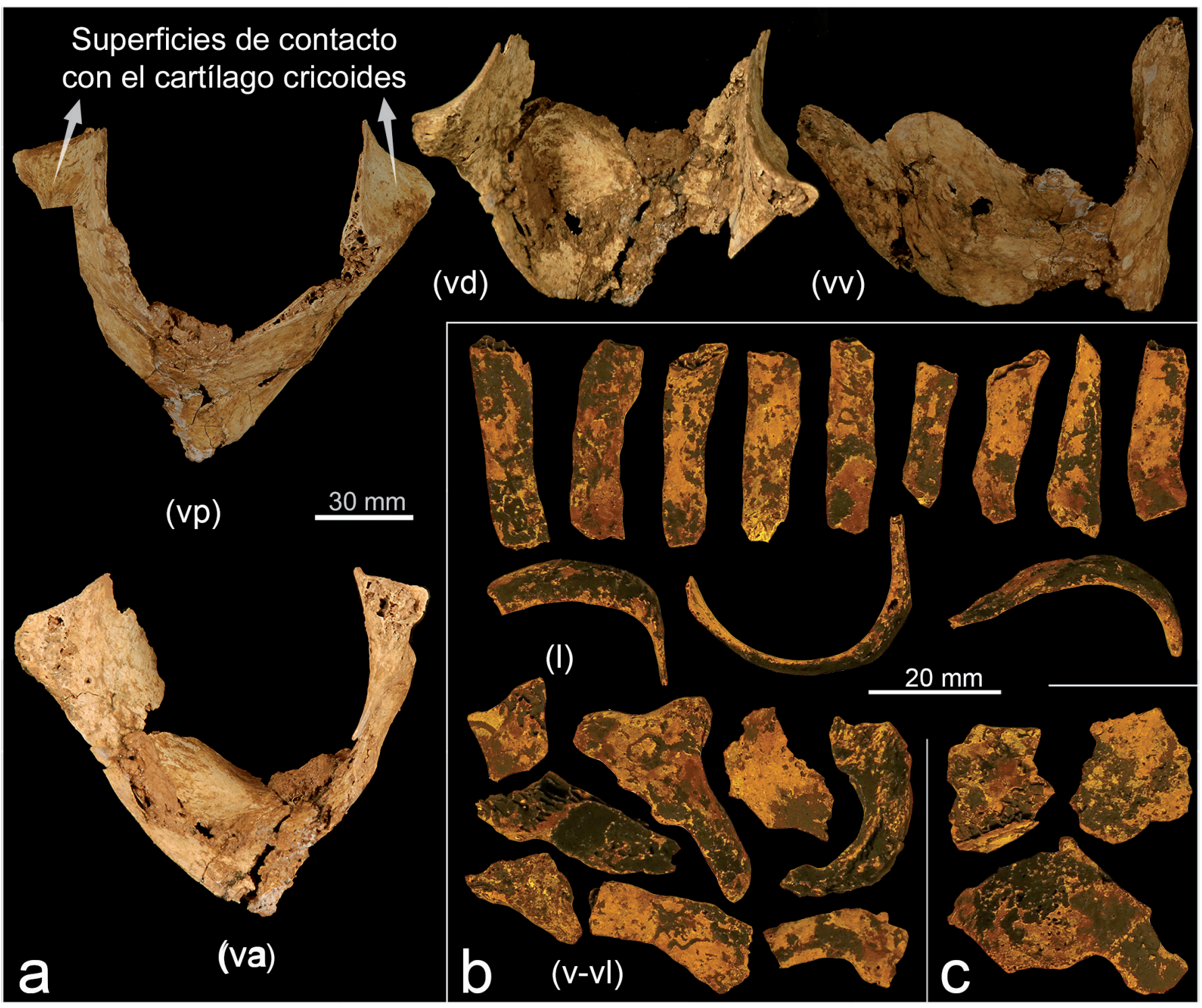

Figura 3. a) Cartílago tiroides de Panochthus sp. (MPLK 0004). b) fragmentos de anillos traqueales de Panochthus sp. (MHM-P 87). c) Fragmentos de cartílago cricoides (MHM-P 87). (1), Fragmentos laterales; (v-vl), fragmentos ventrales y ventrolaterales; (va), vista anterior; (vd), vista dorsal; (vp), vista posterior; (vv), vista ventral.

correspondientes a los anillos traqueales. El cricoides tiene forma similar a la de un anillo traqueal, aunque es de mayor tamaño (Drake et al., 2010) (Fig. 3c). En los mamíferos, este cartílago es completo y sería el único cartílago cerrado (con forma de anillo) de la laringe, asegurando de esta manera que la vía aérea se mantenga abierta en la zona laríngea; a diferencia del cartílago tiroides que se ubica en la parte ventral y los laterales de la laringe (Fig. 4). De los 20 fragmentos de cartílago traqueal, 12 corresponden a segmentos que pertenecen a sectores exclusivamente laterales de los anillos traqueales y varían entre 19 y $54 \mathrm{~mm}$ de largo y entre 6 y $8 \mathrm{~mm}$ de ancho. Los 3 de mayor longitud son curvos (Fig. 3b) y 8 corresponden a porciones ventrales de los anillos. En esta categoría también se incluyen fragmentos que comprenden porciones ventrales y porciones laterales fusionadas (Fig. 3b). Se reconstruyó un anillo traqueal, este anillo pertenecería a una tráquea con forma cilíndrica levemente aplanada en la cara dorsal y ventral, con los extremos libres de cada anillo (en su cara dorsal se solapan, el izquierdo por encima del derecho) (Fig. 4c).

En el cartílago tiroides la longitud dorsoventral máxima (ldvm en Fig. 2a) es de $68 \mathrm{~mm}, 19,5 \mathrm{~mm}$ mayor a la longitud dorsoventral (ldv en Fig. 2a) del anillo traqueal reconstruido. Es preciso aclarar que los extremos libres del cartílago traqueal en los animales vivientes están prácticamente en contacto, ya que el músculo traqueal los mantiene unidos. Sin embargo, en los de ejemplares fósiles, como en Panochthus sp., estos extremos libres se encuentran más separados. En el anillo traqueal la longitud 
transversal (1t en Fig. 2a) es de 53,9 mm, 30,1 mm menos que la longitud transversal máxima (ltm en Fig. 2a) del cartílago tiroides (Figs. 2-4).

\subsection{Músculos relacionados con los cartílagos descriptos}

En Zamorano et al. (2018) se realizó una detallada reconstrucción de los músculos que se insertan o se originan en los huesos del aparato hioides. Dichos músculos forman parte de la musculatura extrínseca de la laringe y se encargan de mover la laringe anterior y posteriormente (ver König \& Liebich, 2005a, 2005b).

Los músculos que discurren entre los cartílagos de la laringe son los denominados músculos intrínsecos de la laringe. Estos son los encargados de ensanchar y estrechar la hendidura glótica y también son responsables de la tensión de los ligamentos vocales (ver König \& Liebich, 2005a). Entre los dos cartílagos laríngeos tratados en esta contribución (tiroides y cricoides), situado lateralmente, se encuentra el músculo cricotiroideo. Este músculo es par, se origina en el borde antero-posterior del cartílago cricoides y se inserta en el margen posterior de la superficie lateral del cartílago tiroides (Fig. 4a). Este último aproxima la parte ventral de los cartílagos cricoides y tiroides. De esta manera, los ligamentos vocales se alargan y tienden a tensarse. Los cricotiroideos, durante la respiración, se encuentran en constante contracción y mantienen la glotis abierta (Sisson \& Grossman, 1982). A su vez, se contraen durante la fonación y se relajan durante la deglución (Negus, 1949).

En Panochthus sp., el área donde se inserta este músculo es amplia y rugosa, lo que provocaría una importante adherencia en la zona de inserción muscular haciendo bascular significativamente el cartílago tiroides hacia el cráneo.

En el sector dorsal de los anillos traqueales se encuentra el músculo traqueal que mantiene unidos los extremos del anillo (Fig. 5b). Su contracción reduce el calibre de la tráquea (vide supra).

\section{DISCUSIÓN Y CONCLUSIONES}

Considerando las medidas expuestas y la relación de tamaño existente entre los cartílagos completos tratados en esta contribución (cartílago tiroides y anillo traqueal) se verifica que las proporciones son las mismas que en otros xenartros, e.g. Myrmecophaga tridactyla Linneo (Naples, 1999).

En síntesis, gracias a condiciones excepcionales de preservación, se conservan tres estructuras de origen cartilaginoso de las vías aéreas: cartílago tiroides, cartílago

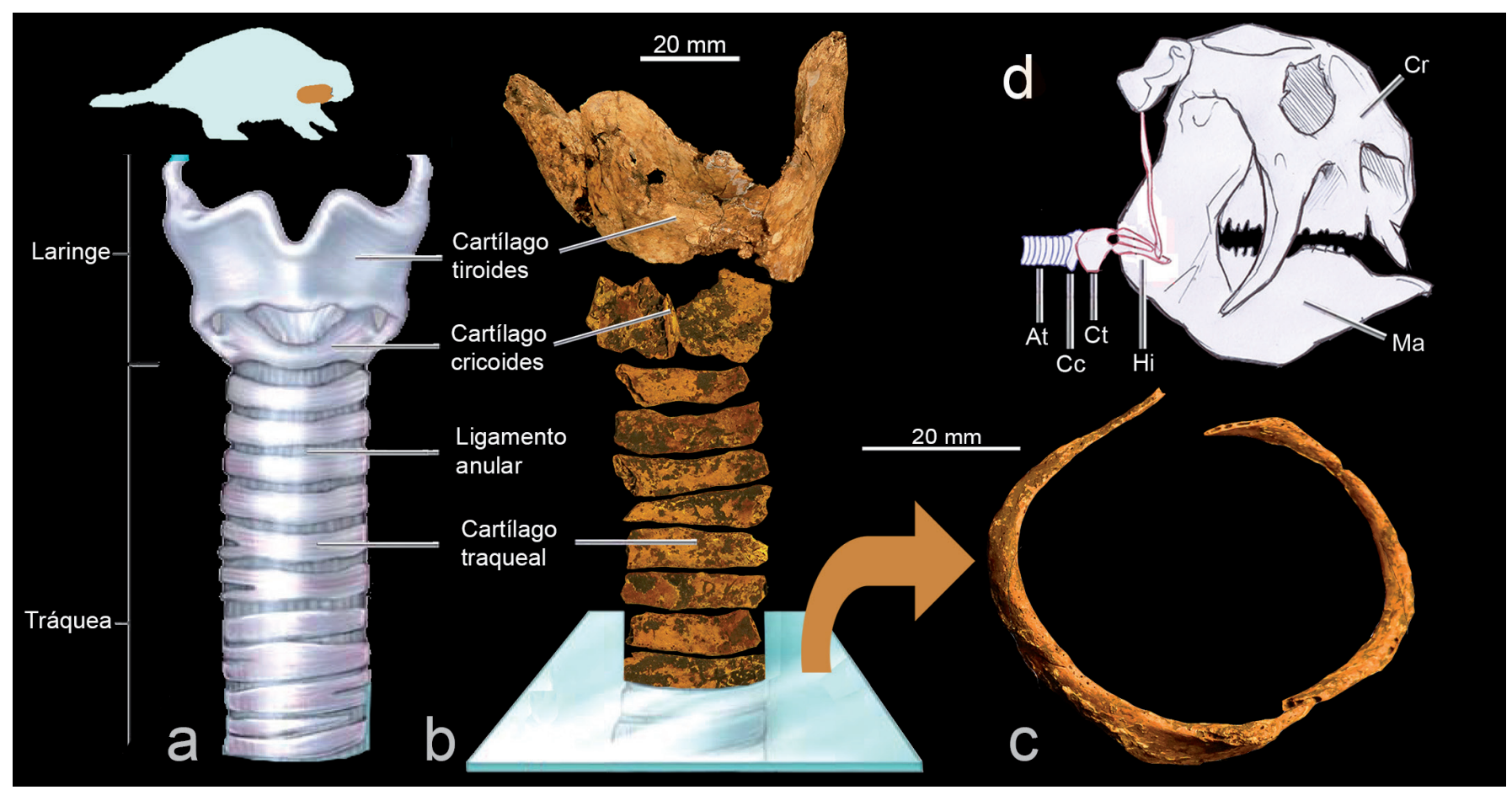

Figura 4. a) Cartílago tiroides, cartílago cricoides y anillos traqueales en un mamífero generalizado. b) Idem que (a) en Panochthus sp. (MPLK 0004 y MHM-P 87). c) Anillo traqueal de Panochthus sp. (MHM-P 87). d) Vista lateral del cráneo de Panochthus, con aparato hioides y cartílagos traqueales. At, anillos traqueales; Cc, cartílago cricoides; Cr, cráneo; Ct, cartílago tiroides; Hi, aparato de hioides; Ma, mandibular. 


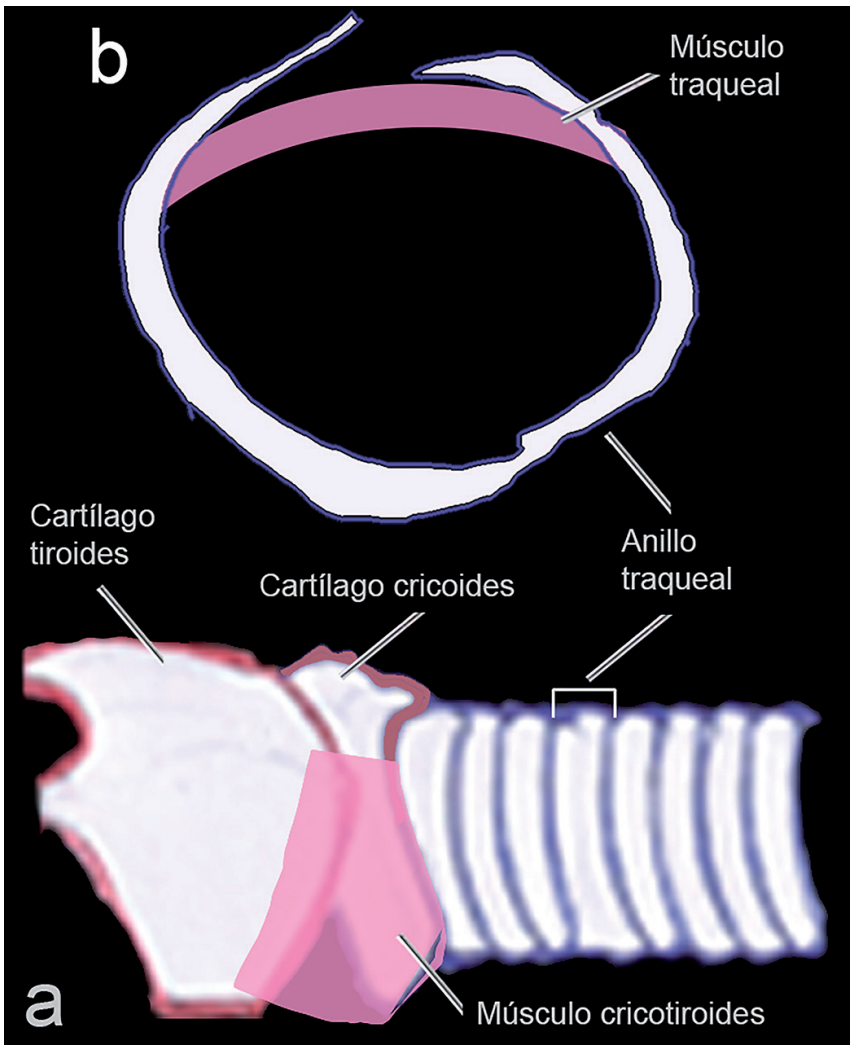

Figura 5. Músculos relacionados con los cartílagos. a) Tiroidescricoides. b) Anillo traqueal.

cricoides y cartílago traqueal (presentes en el cuello) del gliptodonte Panochthus sp., correspondientes a las zonas laríngea y traqueal. Si bien, el cartílago tiroides se conoce en gliptodóntidos, Pérez et al. $(2000,2010)$ lo describen y figuran esquemáticamente para Glyptodon cf. G. clavipes y Zamorano et al. (2018) describen detalladamente y muestran vistas fotográficas de este cartílago en Panochthus sp. Estos últimos trabajos lo hacen en un escenario anatómico sobre el aparato hioides. Del cartílago cricoides y el cartílago traqueal el único registro encontrado para mamíferos fósiles es el del gliptodonte aquí tratado (ver Zamorano, 2020). En la última contribución mencionada se comparan anatómicamente los anillos traqueales con los de animales silvestres y domésticos.

La presente contribución hace referencia a los cartílagos presentes en las vías aéreas fuera del cráneo de un mamífero fósil y, por primera vez, se reconstruyen músculos asociados con estos cartílagos. Se propone que debido a que la superficie rugosa de la lámina lateral del cartílago tiroides, en la cual se inserta el músculo cricotiroides, se produce un mayor anclaje y, por lo tanto, esto generaría un significativo movimiento de este último cartílago. Complementando la propuesta elaborada por Zamorano et al. (2018), realizada exclusivamente con músculos del aparato hioides, el importante desarrollo de desplazamiento del cartílago tiroides posibilitaría que
Panochthus sp. tuviera gran movilidad y, posiblemente, un gran recorrido de la lengua. Futuros estudios como este generarán nuevos conocimientos sobre la paleobiología de la megafauna sudamericana.

\section{AGRADECIMIENTOS}

A Laureano Raúl González Ruíz, Bruno Pianzola y María Julia Sanchez Rondini, por las fotografías. A Juan Cruz González por el dibujo. A Noelia Illarreguy, por la colaboración con el Abstract. A los revisores, Esteban Soibelzon y Sebastián Tambusso, y al editor asociado, Carlos Martínez-Pérez, por enriquecer significativamente la contribución.

\section{REFERENCIAS}

Acuña Navas, M.J., Arce Rodríguez, E., Baquero Barcenas, A.M., Bonilla Mora, W., Coto Chinchilla, K., Guerrero Gamboa, L., Gutiérrez Porras, M., Jiménez Delgado, J., Leitón Villagra, C., Madrigal Rojas, J.P., Monge Carvajal, C., Morales González, F., Núñez Delgado, N., Penón Portmann, M., Quirós Castro, A.G., Rivera Calderón, C. \& Vargas Sanabria, M. 2010. Embriología del desarrollo de los bronquios y el parénquima pulmonar. Medicina Legal de Costa Rica, 27, 61-74.

Ameghino, F. 1889. Contribución al conocimiento de los mamíferos fósiles de la República Argentina. Actas Academia Nacional de Ciencias de Córdoba, 6, 1-1027.

Burmeister, G. 1866. Lista de los mamíferos fósiles del terreno diluviano. Anales del Museo Público de Buenos Aires, 1, 121-232.

Cope, E.D. 1889. The Edentata of North America. The American Naturalist, 23, 657-664.

Drake, R.L., Vogl, A.W. \& Mitchell, A.W. 2010. Gray's Anatomy for Students. 2nd Edition. Churchill Livingstone/ Elsevier, Philadelphia.

Fariña, R.A. 2001. Física y matemáticas para reconstruir la vida en el pasado. Actas de Fisiología, 6, 45-70.

Fernicola, J.C. 2008. Nuevos aportes para la sistemática de los Glyptodontia Ameghino 1889 (Mammalia, Xenarthra, Cingulata). Ameghinana, 45, 553-574.

Gasparini, G.M., Soibelzon, E., Deschamps, C.M., Francia, A., Beilinson, E., Soibelzon, L.H. \& Tonni, E.P. 2016. Continental vertebrates during the Marine Isotope Stage 3 (MIS3) in Argentina. In: Marine Isotope Stage 3 in Southern South America, 60 ka B.P.-30 ka B.P (eds. Gasparini, G.M., Rabassa, J., Deschamps, M.C. \& Tonni, E.P.). Serie Springer Earth System Sciences, 227-247.

Gray, J.E. 1869. Catalogue of Carnivorous, Pachydermatous and Edentate Mammalia in the British Museum. London, British Museum (Natural History). 
Illiger, J.K.W. 1811. Prodromus Systematis Mammalium et Avium Additis Terminis Zoographicis Utriusque Classis. Salfeld: Berolini.

König, H.E. \& Liebich, H.G. 2005a. Anatomía de los Animales Domésticos: Texto y Atlas en Color. Tomo 2: Órganos, Sistema Circulatorio y Sistema Nervioso. Editorial Médica Panamericana. Buenos Aires.

König, H.E. \& Liebich H.G. 2005b. Anatomía de los Animales Domésticos: Texto y Atlas en Color. Tomo 1: Aparato Locomotor. Editorial Médica Panamericana. Buenos Aires.

Naples, V.L. 1999. Morphology, evolution and function of feeding in the giant anteater (Myrmecophaga tridactyla). Journal of Zoology, 249, 19-41; doi: 10.1111/j.14697998.1999.tb01057.x.

Negus, V.E. 1949. La Anatomía y Fisiología Comparadas de la Laringe Hafner, Nueva York.

Owen, R. 1845. Descriptive and Illustrated Catalogue of the Fossil Organic Remains of Mammalia and Aves Contained in the Museum of the Royal College of Surgeons of London. Royal College of Surgeons of London. Museum. London.

Paula Couto, J.C. 1979. Tratado de Paleomastozoología. Academia Brasileira de Ciencias, 1-590.

Pérez, L.M., Scillato-Yané, G.J. \& Vizcaíno, S.F. 2000. Estudio morfofuncional del aparato hioideo de Glyptodon cf. clavipes Owen (Cingulata: Glyptodontidae). Ameghiniana, 37, 293-299.

Pérez, L.M., Toledo, N., De Iuliis, G., Bargo, M.S. \& Vizcaíno, S.F. 2010. Morphology and function of the hyoid apparatus of fossil xenarthrans (mammalia). Journal of Morphology, 271, 1119-1133; doi: 10.1002/jmor.10859.

Scanferla, A., Bonini, R., Pomi, L., Fucks, E. \& Molinari, A. 2013. New Late Pleistocene megafauna assemblage with well-supported chronology from the Pampas of southern South America. Quaternary International, 307, 97-161; doi: 10.1016/j.quaint.2012.08.005.

Scillato-Yané, G.J. 1976. Sobre un Dasypodidae (Mammalia, Xenarthra) de edad Riochiquense (Paleoceno superior) de Itaboraí, Brasil. Anales de la Academia Brasilera de Ciências, 48, 529-530.

Scillato-Yané, G.J. 1977. Sur quelques Glyptodontidae nouveaux (Mammalia, Edentata) du Déséadien (Oligoce sus ne inferieur) de Patagonie (Argentine). Bulletin $d u$ Muséum National d'Histoire Naturelle, 64, 249-262.

Scillato-Yané, G.J. \& Carlini, A.A. 1995. Estado actual del estudio de los Xenarthra. Id., Simposio "Estado actual del estudio de los Xenarthra". Abstract Book, p. 27.

Scillato-Yané, G.J. \& Carlini, A.A. 1998. Un gigantesco gliptodonte en los alrededores de la ciudad de La Plata. Revista Museo de La Plata, 11, 45-48.

Sisson, S. \& Grossman, J.D. 1959. Anatomía de los Animales Domésticos. $5^{\mathrm{a}}$ edición. Salvat, Barcelona.

Sisson, S. \& Grossman, J.D. 1982. Anatomía de los Animales Domésticos: $1^{a}$ Parte. Salvat, Barcelona.
Soibelzon, L.H., Zamorano, M., Scillato-Yané, G.J., Piazza, D., Rodríguez, S., Soibelzon, E., Tonni, E.P., San Cristóbal, J. \& Beilinson, E. 2012. Un Glyptodontidae de gran tamaño en el Holoceno temprano de la región Pampeana. Revista Brasileira de Paleontologia, 15, 113-122.

Zamorano, M. 2012. Los Panochthini (Xenarthra, Glyptodontidae): sistemática y evolución. Ph.D. Thesis, Universidad Nacional de la Plata (unpublished).

Zamorano, M. 2013. Diagnosis y nueva descripción de Propanochthus bullifer (Burmeister) (Xenarthra, Glyptodontidae). Consideraciones bioestratigráficas y cronológicas de su procedencia). Spanish Journal of Palaeontology, 28, 283-292.

Zamorano, M. 2020. Exceptional preservation of tracheal rings in a fossil mammal: the case of Panochthus sp. (Xenarthra, Glyptodontidae). Acta Palaeontologica Polonica, 65, 29-34; doi: 10.4202/app.00654.2019.

Zamorano, M. \& Jara Almonte, G. 2018. Primer registro fehaciente de Panochthus (Xenarthra; Cingulata; ptodontidae) para Perú. Acta Geológica Lilloana, 30, 23-30.

Zamorano, M., Scillato-Yane, G.J. \& Zurita, A.E. 2014a. Revisión del género Panochthus (Xenarthra, Glyptodontidae). Revista del Museo de La Plata (Sección Paleontología), 14, 1-46.

Zamorano, M., Taglioretti, M., Zurita, A.E., Scillato-Yané, G.J. \& Scaglia, F. 2014b. El registro más antiguo de Panochthus (Xenarthra, Glyptodontidae). Estudios Geológicos, 70, 5-11; doi: 10.3989/egeol.41443.289.

Zamorano, M., Scillato-Yané, G.J., Soibelzon, E., Soibelzon, L.H., Bonini, R. \& Rodriguez, S. 2018. Hyoid apparatus of Panochthus sp. (Xenarthra; Glyptodontidae) from the Late Pleistocene of the Pampean region (Argentina). Comparative description and muscle reconstruction. Neues Jahrbuch für Geologie und Paläontologie, 288, 205-219; doi: 10.1127/njgpa/2018/0733.

Zurita, A.E., Miño-Boilini, A.R., Soibelzon, E., Carlini, A.A. \& Paredes-Ríos, F. 2009. The diversity of Glyptodontidae (Xenarthra, Cingulata) in the Tarija Valley (Bolivia) systematic, biostratigraphic and paleobiogeographic aspects of a particular assemblage. Neues Jahrbuch für Geologie und Paläontologie, 251, 225-237; doi: 10.1127/0077-7749/2009/0251-0225.

Zurita, A.E., Scillato-Yané, G.J., Ciancio, M., Zamorano, M. \& González Ruiz, L.R. 2016. Los Glyptodontidae (Mammalia, Xenarthra): historia biogeográfica y evolutiva de un grupo particular de mamíferos acorazados. In: Historia Evolutiva y Paleobiogeográfica de los Vertebrados de América del Sur (eds. Agnolin, F.L., Lio, G.L., Brisson Egli, F., Chimento, N.R. \& Novas F.E.). XXX Jornadas Argentinas de Paleontología de Vertebrados. Contribuciones del MACN, 6, 249-262. 
